Provided for non-commercial research and education use. Not for reproduction, distribution or commercial use.
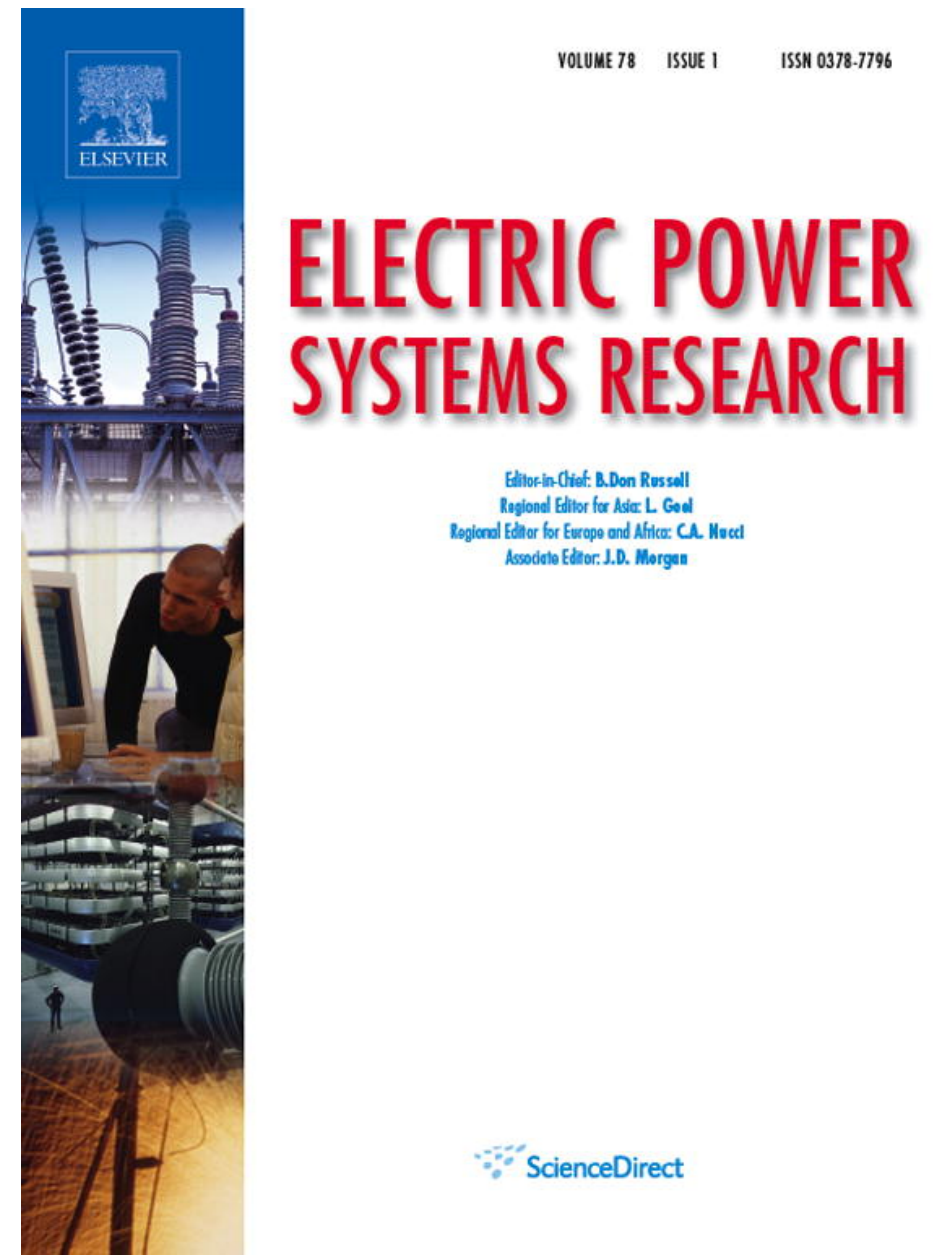

\footnotetext{
Ehatiorinchiet: B.Don Russoll

Ragional Elitior for Asic L Gool

Rogiconl Edhor for Europo and Afice: CC. Meecl

Assodiale Edertor. J.D. Morgen
}

This article was published in an Elsevier journal. The attached copy

is furnished to the author for non-commercial research and education use, including for instruction at the author's institution, sharing with colleagues and providing to institution administration.

Other uses, including reproduction and distribution, or selling or licensing copies, or posting to personal, institutional or third party websites are prohibited.

In most cases authors are permitted to post their version of the article (e.g. in Word or Tex form) to their personal website or institutional repository. Authors requiring further information regarding Elsevier's archiving and manuscript policies are encouraged to visit: 


\title{
Active/reactive bid based dispatch models to be used in electricity markets
}

\author{
Mário Helder Rodrigues Gomes ${ }^{\mathrm{a}}$, João Tomé Saraiva ${ }^{\mathrm{b}, *}$ \\ ${ }^{a}$ Departamento de Engenharia Electrotécnica, Escola Superior de Tecnologia de Tomar, Quinta do Contador, Estrada da Serra, 2300 Tomar, Portugal \\ b INESC Porto and Departamento de Engenharia Electrotécnica e Computadores, Faculdade de Engenharia da Universidade do Porto, \\ Campus da FEUP, Rua Dr. Roberto Frias, 4200 - 465 Porto, Portugal
}

Received 26 April 2006; received in revised form 12 December 2006; accepted 2 January 2007

Available online 12 February 2007

\begin{abstract}
This paper describes two new active/reactive dispatch models to be used by System Operators in order to assign reactive power and to validate the economic schedules prepared by Market Operators together with the injections related with Bilateral Contracts. When talking about electricity markets one usually refers to active power markets paying less attention to ancillary services, namely to reactive power/voltage control. This usually leads to a chronological sequence of activities that may lead to inefficiencies because active and reactive powers are coupled given the capability diagram of synchronous generators, the ac power flow equations and the branch thermal limits. In this paper, we propose new models to remarry active and reactive allocation procedures based on a market approach as a way to ensure operation transparency. The resulting optimization problems are solved by a Sequential Linear Programming, SLP, approach that allows one to compute active and reactive nodal marginal prices at its final iteration. The paper includes a case study based on the IEEE 24 Bus Test System to illustrate the application of the developed models and demonstrate their interest in the scope of restructured power systems.
\end{abstract}

(C) 2007 Elsevier B.V. All rights reserved.

Keywords: Integrated active/reactive dispatch; Electricity markets; System Operator; Active/reactive nodal marginal prices; Sequential linear programming

\section{Introduction}

The restructuring of traditional power utilities to introduce competitive mechanisms in the sector started in Chile in the early 1980th, spread to England and Wales and then to other European countries, USA, Latin America, Australia and New Zealand. When completing two decades after its beginning, it is possible to recognize that there are a number of common features to all these movements despite the peculiarities of each particular implementation. In a first step, vertically integrated utilities were unbundled in generation companies, transmission providers and distribution entities. Then, the eligibility level was progressively decreased and distribution companies were decoupled in wiring distribution providers and in retailers. For instance, in the EU countries this separation was enforced under the EU 2003/54/EC Directive Establishing the Common Rules for the Internal Electricity Market [1]. Integrated tariffs were also separated to better follow this new structure in terms of pricing

\footnotetext{
* Corresponding author. Tel.: +351 22 2094230; fax: +351222094150.

E-mail addresses: mgomes@ipt.pt (M.H.R. Gomes), jsaraiva@ fe.up.pt (J.T. Saraiva).
}

the services provided by different agents, to allocate costs adequately and to eliminate cross-subsidies. Apart from these new corporative and tariff structures, operation planning was also greatly modified since the supply and the demand relate themselves by day-ahead markets and by Bilateral Contracts and the operation planning functions are now typically split between a Market Operator and a System Operator.

It is important to recognize that electricity is not an easily marketable product or, in other words, it is far from being a true commodity. It is not possible to store it in large quantities, it must be produced at the same time it is consumed, physical laws determine power system operation and there is a network that often prevents the implementation of optimal economic generation strategies. Apart from that, in most countries the technical characteristics and technologies in the generation mix are very different, the time lag between the decision to build an asset and its commissioning is usually large and finally the demand still displays a very inelastic behavior to price changes. When talking about electricity markets, it is also important to recognize that one usually refers to active power markets, neglecting for instance reactive power/voltage control. This lack of attention paid to reactive power is explained because it is not so directly priced as active power and, in any case, there is the idea that 
its price is much more reduced when compared with the one of active power. However, active and reactive powers are inherently married for a large number of reasons, namely:

- the operation of synchronous generators is determined by capability PQ diagrams. This means that a reactive power requirement issued by the System Operator may be unfeasible given the active power already scheduled by the Market Operator. If the active output is reduced, the income of that generator will decrease regarding the value expected from the daily market, leading to what is known as an opportunity cost;

- secondly, active and reactive powers are coupled in terms of the ac power flow equations and both of them lead to the branch flows used to evaluate branch thermal limits;

- finally, reactive power is closely linked with voltage control and their local nature is well known as well as their importance to ensure the secure operation of power systems. This means that in several cases more costly bids originally not accepted in the day-ahead market may have to be used to enforce branch limits or to alleviate voltage constraints.

In this paper we recognize the coupling between active and reactive power and that several market implementations are based on a sequence of activities that may not reflect in an adequate way the characteristics of electricity. In view of this, this paper presents an approach to remarry to a certain extent active and reactive powers while retaining competitive aspects. This approach is based on the economic dispatch prepared by the Market Operator together with the Bilateral Contract injections. The System Operator evaluates this set of injections and if they lead to an unfeasible operation point, it uses adjustment supply and demand bids to identify a new dispatch that minimizes branch active losses together with the cost of these adjustments. This strategy aims at ensuring that the final active dispatch is as close as possible regarding the initial one. This means that the initial active injections will only be changed if that is required from a technical point of view thus contributing to ensure the transparency of the whole procedure. The results include the final active and reactive dispatch, the generator adjustments required to enforce voltage or branch limit constraints, the generator adjustments needed to balance branch active losses and the nodal active and reactive marginal prices related with dual variables of several constraints of the optimization problem. The approach described in this paper enlarges and completes the one presented in [2]. In the first place, we decoupled generator adjustment variables in two terms allowing the allocation of branch losses to each generator and then we generalized the formulation since we are now considering both the Market Operator initial schedule and the Bilateral Contract injections.

Apart from this introduction, Section 2 describes several reactive power allocation approaches described in the literature and Section 3 describes in detail the mathematical formulation of the problem including aspects as the adjustment bids and the modeling of the synchronous generator capability diagram. Section 4 details the application of Sequential Linear Programming, SLP, to solve the resulting non-linear optimization problem and the computation of the active and reactive nodal marginal prices.
Section 5 presents a case study based on the IEEE 24 Bus Test System and finally Section 6 presents the most relevant conclusions.

\section{Reactive power allocation approaches}

Under the traditional paradigm, reactive power/voltage control was managed in a centralized way inside the vertically integrated utilities. With the advent of Independent Generation, several promoters established long-term Power Purchase Agreements, PPA, regulating the operation and the remuneration of their stations. These PPA's typically included rules for the provision of what is now known as ancillary services. In any case, the attention given to reactive power was typically much reduced and this was in line with its reduced economic impact when compared with the cost of generating active power. This more reduced attention was also certainly due to the fact that the cost of providing active power is established in a much more direct way when compared with the traditional difficulty of assigning a cost to reactive power. However, this insufficient attention is not in line with the importance of reactive power when addressing voltage control and system security.

With the advent of electricity markets, reactive power started to receive more attention reflected in a larger number of publications as [3-19]. In many systems reactive power was considered as a mandatory non-paid service that should be provided by all generators participating in the market. It was only afterwards that regulations started to be modified to design competitive mechanisms and to implement models to price reactive support. As an example, reference [3] describes the provisions in force in England and Wales after the privatization of the industry and it proposes a mechanism to price reactive power based on utilization and capability costs designed to create a reactive power market. The authors described an OPF model to assign reactive power based on linearized reactive power cost curves offered by individual generators. This type of problem is also addressed in [4] now considering an objective function that includes terms reflecting the cost of adjusting control devices in order to reduce the number of control changes. Reference [5] also penalizes the number of control actions required to bring the system to a different operation point and it models some constraints in a soft way adopting Fuzzy Set concepts.

Reference [6] proposes pricing reactive power according to the availability of reactive power capacity since variable reactive power generation costs are difficult to evaluate. In order to price this capacity the authors suggest the use of a percentage of generator costs or the use of synchronous condenser costs. As for the allocation of these costs to network users, the authors suggest the adoption of demand performance requirements defining the limit of absorption of reactive power regarding active power without cost or the implementation of local reactive power markets leading to the computation of nodal or zonal reactive power locational values.

The use of locational values is further developed in references $[7,8]$. In the first of them, the authors obtain nodal marginal reactive prices as a sub-product of an optimization problem that 
minimizes active power generation costs and active transmission losses while reference [8] uses zonal prices related to each control area.

Regarding the value of reactive power and given the capability diagram of a generator, references [9-11] define the opportunity cost as the cost incurred by a generator if a particular reactive power requirement can only be accomplished if the prescheduled active power is changed. This reduction of the active output leads to a reduction of the generator revenue and this value can then be used to price reactive power.

References [12,13] describe multi-objective operation planning models recognizing the contradictory nature of several objectives. In [12], the model considers the minimization of transmission losses and the maximization of a stability index and [13] describes a bid based active/reactive model using active and reactive generation costs based on generator bids, active transmission losses and an index measuring the quality of the voltage profile.

References [14,15] organize the reactive power payments in terms of a capability and an utilization term [14] and in terms of a fixed and a variable cost [15]. The OPF model in [14] is based on generator offers while the variable costs in [15] are directly related with the opportunity costs referred above. Reference [16] describes a reactive power bid structure that directly uses the concept of opportunity cost. According to this structure, there is an amount of reactive power for which the generator will not be paid. Then, there is an amount of reactive power that can be provided without changing the active output and finally above this range, a reactive requirement can only be fulfilled if one reduces the active power and the corresponding revenue. A similar structure is also used in $[17,18]$ to allocate reactive power while enforcing the ac power flow equations, branch flow and voltage limit constraints as well as generator limit constraints.

Finally, reference [19] details the activities developed in the Spanish electricity market, namely the ones of the Transmission System Operator, TSO. The authors describe a two-stage model to enforce branch and voltage constraints and to allocate reactive power. The first stage is designed to get a feasible solution by performing a redispatch and adjusting voltage control devices while minimizing the total system cost. In the second stage, the voltage control devices are fine tuned by minimizing active transmission losses.

\section{Active/reactive power dispatch using adjustment bids}

\subsection{General issues}

Before detailing the two optimization models developed to validate the schedule prepared by the Market Operator together with the injections associated to Bilateral Contracts, we will address a number of concepts and models used in these formulations. They include the uniform price auction run by the Market Operator, the capability diagram of synchronous generators, and the adjustment bids communicated both by generators and loads to the System Operator.

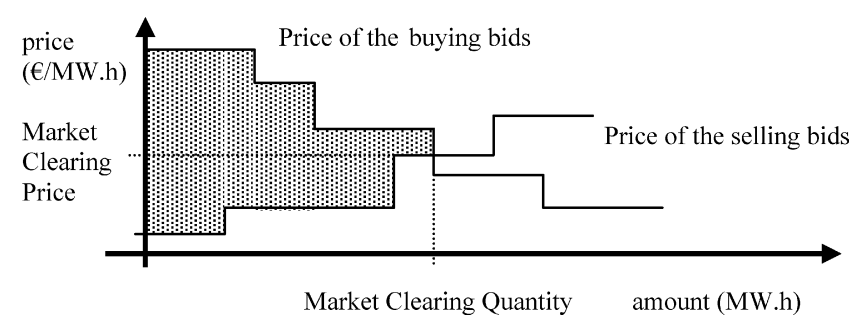

Fig. 1. Matching process in an uniform price auction.

\subsection{Market Operator initial uniform price auction}

Typical electricity markets are organized in terms of a set of activities that are usually assigned to different entities. In day $n-1$ the Market Operator receives selling and buying bids from market agents that, in their simplest version - Simple Bids - include pairs (quantity, price). The Market Operator orders selling bids by the ascending order of its price and buying bids in descending order of the corresponding price so that they are built the aggregated generation and demand curves in Fig. 1. The intersection of these two curves leads to the Clearing Quantity and to the Clearing Price, interpreted as the short-term marginal price of the generation system.

This problem can be modeled by (1)-(4). In this formulation $\mathrm{Cd}_{j}$ and $\mathrm{Cg}_{i}$ are the buying and selling prices, $\mathrm{Pd}_{j}^{\text {bid }}$ and $\mathrm{Pg}_{i}^{\text {bid }}$ the maximum demand and generation bid quantities, $\mathrm{Pd}_{i}$ and $\mathrm{Pg}_{i}$ are the demand and generation at the final solution and $\mathrm{Nd}$ and $\mathrm{Ng}$ are the number of buying and selling bids. The objective function $Z$ in (1) corresponds to the Social Welfare Function, and it represents the surplus between the aggregated demand and generation curves, that is the shaded area in Fig. 1. This objective function is subjected to limits on the demand (2) and on the generation (3) and to a demand/supply balance Eq. (4).

$\max Z=\sum_{j=1}^{\mathrm{Nd}} \mathrm{Cd}_{j} \cdot \mathrm{Pd}_{j}-\sum_{i=1}^{\mathrm{Ng}} \mathrm{Cg}_{i} \cdot \mathrm{Pg}_{i}$

subject to $0 \leq \operatorname{Pd}_{j} \leq \operatorname{Pd}_{j}^{\text {bid }}$

$0 \leq \mathrm{Pg}_{i} \leq \mathrm{Pg}_{i}^{\text {bid }}$

$\sum_{j=1}^{\mathrm{Nd}} \mathrm{Pd}_{j}=\sum_{i=1}^{\mathrm{Ng}} \mathrm{Pg}_{i}$

Problem (1)-(4) uses simple bids and it corresponds to a single step formulation that can be enhanced in several ways. In the first place, generators can be allowed to transmit to the Market Operator bids structured in a number of blocks as sketched in Fig. 2. This allows bids to better follow the generators' cost curve and eventually the first block can be declared indivisible. This would mean that, if scheduled by the Market Operator, the first block should be entirely dispatched, namely as a way to cope with minimum technical limits of thermal generators. Secondly, generators can also include in their bids other information that transforms the initially independent $24 \mathrm{~h}$ schedules into a single coupled problem. This would mean passing from 


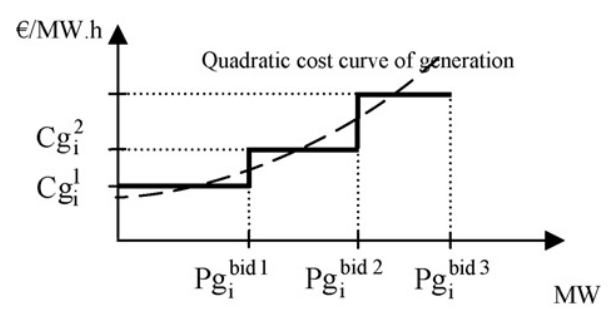

Fig. 2. Block structure of selling bids.

the referred Simple Bids to Complex Bids, and this information can correspond to up and down ramps, for instance.

In the developed models described in this paper we used simple selling bids having block structure. This means that generators transmit to the Market Operator information about blocks and the corresponding selling prices and the demand communicates bids including powers and buying prices.

\subsection{Synchronous generator capability diagram}

The operation of synchronous generators is characterized by coupled active/reactive power diagrams as the one represented in Fig. 3. This diagram delimits the generator feasible operation points in the PQ plan. The limiting curve is in fact due to several constraints that determine the operation of synchronous generators. In our case, we modeled the capability diagram of Fig. 3 using the following three curves:

- curve 1, between $\mathrm{Qg}_{i}^{\max }$ and $\underline{S}_{1}$, represents the rotor field current limit;

- curve 2, from $\underline{S}_{1}$ to $\underline{S}_{2}$, is the armature limit;

- curve 3, the arc between $\mathrm{Qg}_{i}^{\min }$ and $\underline{S}_{2}$, represents the stability limit.

These curves can be approximated by linear expressions, as it will be used in Section 3.6. Given this diagram, it is important to realize that a reactive power requirement can be unfeasible, even if both the $P$ and the $Q$ individual and separate limits are not violated. This fact is important because the Market Operator can assign a particular generator a $P$ schedule that turns unfeasible the $Q$ requirement issued afterwards by the System Operator.

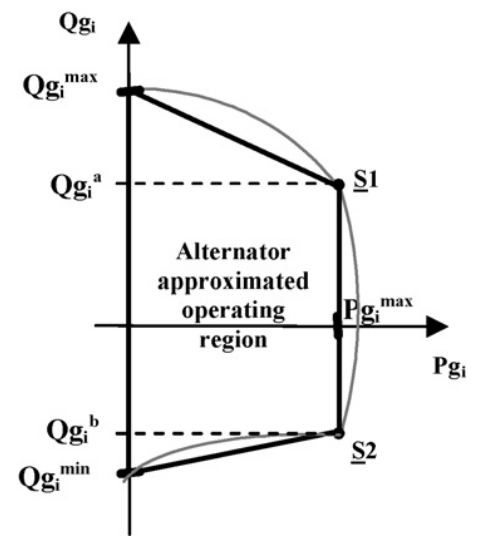

Fig. 3. Capability diagram of a synchronous generator.
This problem is even more relevant given that reactive power has a well-known local nature and in some cases a particular generator must in fact provide reactive power due to voltage considerations and to its particular location in the network. In this case, if the active power output regarding the Market Operator schedule has to be reduced, then the expected revenue of that generator is also reduced. This corresponds to the opportunity cost referred in Section 2 and it becomes obvious that electricity markets should be designed in a way to provide some sort of compensation in such cases.

In the developed models, the System Operator must know the data characterizing the capability diagram of each generator so that it is possible to formulate equations for the curves referred above. This includes the values of $\mathrm{Pg}_{i}^{\max }$, $\mathrm{Qg}_{i}^{\max }, \mathrm{Qg}_{i}^{\min }, \mathrm{Qg}_{i}^{\mathrm{a}}$ and $\mathrm{Qg}_{i}^{\mathrm{b}}$.

\subsection{Generator adjustment bids}

The developed models use the economic schedule prepared by the Market Operator together with the injections from Bilateral Contracts as the initial information to be validated by the System Operator. In view of this information, if no operation or security constraint is violated this set of Market Operator and Bilateral Contract injections are technically feasible. However, they may have to be changed if there is congestion or if voltage limit constraints are violated. In this case, the System Operator activates a secondary market, an Adjustment Market, that uses adjustment bids both from generators and loads. This way, it will be possible to conduct this technical study in a transparent way because it is based on a competitive market mechanism.

Generator adjustment bids are sent to the System Operator and include the acceptable maximum variation, $\mathrm{vg}_{i}^{\text {tol }}$, that the Market Operator based schedule or the Bilateral Contract generations can suffer together with the adjustment price, $\mathrm{Cg}_{i}^{\mathrm{A}}$.

In case a generator was not originally dispatched, its maximum possible adjustment can correspond to a percentage of its installed capacity. If this generator is required by the System Operator to help solving some system constraint its revenue will then correspond to the product of the adjustment price by the allocated power meaning that it is not explicitly present any fixed start up cost. In order not to destroy the continuity of the problem, such a cost could be diluted in the value of the offered adjustment price. Alternatively, considering an explicit start up cost would require the use of binary variables and more time-consuming solution techniques.

\subsection{Demand adjustment bids}

The regulatory framework in force in several power systems already includes the possibility of reducing load if that is required to ensure the security of the system. These mechanisms are known as interruptible contracts and they include some sort of compensation for loads that admit providing this service. This concept is based on the fact that power system constraints can be alleviated not only by redispatching generation but also in some cases if one admits load adjustments. Using this concept, load adjustment bids include information regarding 
the maximum reduction a load admits to be curtailed regarding the amount scheduled by the Market Operator or related to a Bilateral Contract, as well as the corresponding adjustment price.

This information is sent to the System Operator so that this entity knows all the available resources of this nature. The System Operator will then eventually accept some of them depending on the violated constraints and on the relation of prices included in the adjustment bids. This certainly increases the flexibility that the System Operator has to regain feasibility apart from increasing the competition and liquidity of this secondary adjustment market.

\subsection{Mathematical formulation-Model 1 (unclosed mechanism)}

In the first model we admit that there are two contractual electricity systems sharing the same grid. These two contractual systems are the Market Operator and the Physical Bilateral Contracts. The generators and loads that presented bids to the Market Operator are then organized in two groups because some of them were dispatched and some others were not, depending on their biding prices. Accordingly, in this formulation we classified generators and loads in three sets as follows:

- $S_{1}$-it includes generators and loads that presented bids to the Market Operator and that were dispatched in the day-ahead market;

- $S_{2}$-it includes generators and loads that were not dispatched in the day-ahead market although they presented bids to the Market Operator;

- $S_{3}$-it includes generators and loads that have physical Bilateral Contracts.

The formulation to be described will be used by the System Operator to allocate the active power required to balance active losses, to allocate reactive power, and to change the active power initially scheduled by the Market Operator or associated to Bilateral Contracts in order to solve system constraints. Therefore, the formulation considers two types of active power adjustment variables:

- the first one is related with changes on the initially scheduled active powers required to balance active power transmission losses. These variables correspond to $\Delta \mathrm{Pg}_{i}^{\mathrm{L}}$ and the index $i$ belongs to sets $S_{1}$ and $S_{3}$. In a more general situation, we can also admit that a generator included in set $S_{2}$ is asked by the System Operator to contribute to balance active losses if that helps to minimize transmission losses;

- the second one corresponds to changes on the initially scheduled generation and load active powers required to solve system constraints (for instance, to ensure voltage magnitudes or power flow thermal limits). They can also correspond to an active power generation reduction requested by the System Operator so that a generator can provide a certain level of reactive power support according to its capability diagram. These variables are represented by $\Delta \mathrm{Pg}_{i}^{\mathrm{A}}$ and by $\Delta \mathrm{Pd}_{i}^{\mathrm{A}}$ for generation and load adjustments and the index $i$ takes values in the sets $S_{1}, S_{2}$ and $S_{3}$ for generation variables and in sets $S_{1}$ and $S_{3}$ for load variables.

Considering these notation aspects, the objective function of the problem (5) integrates the following five terms:

- the first one is related with the amount of active power assigned to each generator in order to balance active transmission losses. As referred before, the associated adjustment variables are $\Delta \mathrm{Pg}_{i}^{\mathrm{L}}$ where the index $i$ stands for generators dispatched or not by the Market Operator as well as generators having Bilateral Contracts. In this formulation we used the Market Operator clearing price, $\rho^{\mathrm{MO}}$, to remunerate generators for loss compensation. This corresponds to a market design aspect and different remuneration alternatives could be implemented including, for instance, the use of the adjustment generation price referred in Section 3.4. In any case, there are systems that remunerate the generation of active losses on a marginal price basis. For instance, Norwegian distribution companies have to buy the active power to balance network losses in the dayahead market meaning that generators will then be paid on a marginal basis. In a similar way, the Spanish System Operator computes hourly nodal active loss sensitivity coefficients once the daily Market Operator dispatch and the Bilateral Contract powers are known. These coefficients are then multiplied by the dispatched quantities in order to get the real generation amounts. This approach leads to a result that is similar to the one obtained if we directly use the Market Operator clearing price, $\rho^{\mathrm{MO}}$, as adopted in the models described in this paper;

- the second and the third terms represent the adjustment costs both for generators and for loads that bided in the day-ahead market. The two corresponding summations are computed for generators in sets $S_{1}$ and $S_{2}$ and for loads in set $S_{1}$;

- the fourth and the fifth terms are related with the injections from Bilateral Contracts and represent the corresponding generator and load adjustment costs. These two summations are computed for generators and loads in set $S_{3}$.

$$
\begin{aligned}
\min Z= & \rho^{\mathrm{MO}} \cdot \sum_{S_{1} \cup S_{2} \cup S_{3}} \Delta \mathrm{Pg}_{i}^{\mathrm{L}}+\sum_{S_{1} \cup S_{2}}\left|\Delta \mathrm{Pg}_{i}^{\mathrm{A}}\right| \times \mathrm{Cg}_{i}^{\mathrm{A}} \\
& +\sum_{S_{1}}\left|\Delta \mathrm{Pd}_{j}^{\mathrm{A}}\right| \times \mathrm{Cd}_{j}^{\mathrm{A}}+\sum_{S_{3}}\left|\Delta \mathrm{Pg}_{i}^{\mathrm{A}}\right| \times \mathrm{Cg}_{i}^{\mathrm{A}} \\
& +\sum_{S 3}\left|\Delta \mathrm{Pd}_{j}^{\mathrm{A}}\right| \times \mathrm{Cd}_{j}^{\mathrm{A}}
\end{aligned}
$$

Subj. $\quad \Delta V_{i}^{\min } \leq \Delta V_{i} \leq \Delta V_{i}^{\max }, \quad$ for all nodes

$\Delta \theta_{i j}^{\min } \leq \Delta \theta_{i j} \leq \Delta \theta_{i j}^{\max }, \quad$ for all nodes

$0 \leq \Delta \mathrm{Pg}_{i}^{\mathrm{L}} \leq \Delta \mathrm{Pg}_{i}^{\mathrm{L} m a x}, \quad$ for $i \in S_{1} \cup S_{2} \cup S_{3}$

$-\frac{\mathrm{vg}_{i}^{\text {tol }}}{100} \times \operatorname{Pg}_{i} \leq \Delta \mathrm{Pg}_{i}^{\mathrm{A}} \leq \frac{\mathrm{vg}_{i}^{\text {tol }}}{100} \times \operatorname{Pg}_{i}, \quad$ for $i \in S_{1} \cup S_{3}$ 
$0 \leq \Delta \mathrm{Pg}_{i}^{\mathrm{A}} \leq \frac{\operatorname{vg}_{i}^{\mathrm{A}}}{100} \times \mathrm{Pg}_{i}^{\max }, \quad$ for $i \in S_{2}$

$\Delta \mathrm{Pg}_{i}^{\min } \leq \Delta \mathrm{Pg}_{i}^{\mathrm{A}}+\Delta \mathrm{Pg}_{i}^{\mathrm{L}} \leq \Delta \mathrm{Pg}_{i}^{\max }, \quad$ for $i \in S_{1} \cup S_{2} \cup S_{3}$

$-\mathrm{Pd}_{j} \leq \Delta \mathrm{Pd}_{j}^{\mathrm{A}} \leq 0, \quad$ for $i \in S_{1} \cup S_{3}$

$\mathrm{Qg}_{i}+\Delta \mathrm{Qg}_{i} \leq \mathrm{Qg}_{i}^{\mathrm{max}}-\frac{\mathrm{Qg}_{i}^{\max }-\mathrm{Qg}_{i}^{\mathrm{a}}}{\mathrm{Pg}_{i}^{\max }} \cdot\left(\mathrm{Pg}_{i}+\Delta \mathrm{Pg}_{i}^{\mathrm{A}}+\Delta \mathrm{Pg}_{i}^{\mathrm{L}}\right)$,

for $i \in S_{1} \cup S_{2} \cup S_{3}$

$\mathrm{Qg}_{i}+\Delta \mathrm{Qg}_{i} \geq \mathrm{Qg}_{i}^{\mathrm{min}}+\frac{\mathrm{Qg}_{i}^{\mathrm{b}}-\mathrm{Qg}_{i}^{\min }}{\mathrm{Pg}_{i}^{\max }} \cdot\left(\mathrm{Pg}_{i}+\Delta \mathrm{Pg}_{i}^{\mathrm{A}}+\Delta \mathrm{Pg}_{i}^{\mathrm{L}}\right)$,

for $i \in S_{1} \cup S_{2} \cup S_{3}$

$\Delta S_{i j}^{\min } \leq \Delta S_{i j}(\Delta V, \Delta \theta) \leq \Delta S_{i j}^{\max }, \quad$ for all branches

$\sum_{k=1}^{\mathrm{Nb}} \Delta P_{k}^{\text {loss }}(\Delta V, \Delta \theta)=\sum_{S_{1} \cup S_{2} \cup S_{3}} \Delta \mathrm{Pg}_{i}^{\mathrm{L}}$

$\Delta P_{i}^{\mathrm{inj}}(\Delta V, \Delta \theta)=\left(\Delta \mathrm{Pg}_{i}^{\mathrm{A}}+\Delta \mathrm{Pg}_{i}^{\mathrm{L}}\right)-\Delta \mathrm{Pd}_{i}^{\mathrm{A}}, \quad$ for all nodes

$\Delta Q_{i}^{\mathrm{inj}}(\Delta V, \Delta \theta)=\Delta \mathrm{Qg}_{i}-\Delta \mathrm{Qd}_{i}, \quad$ for all nodes

The objective function (5) is subjected to several constraints as follows:

- constraints (6) and (7) are related with bounds on nodal voltages and on branch phase differences. Regarding nodal voltages, Grid Operation Manuals publicly available in several countries usually give indications regarding the allowable range for each voltage level. The minimum and maximum voltage deviation limits in constraint (6) are computed regarding these allowable ranges. These constraints together with the minimization of branch active losses ensure that the final solution has an adequate voltage profile;

- constraints (8) impose bounds on the adjustment generation variables related with losses, both for the generators that presented bids to the Market Operator and for Bilateral Contract generators;

- constraints (9) impose bounds on the generation adjustments in terms of a maximum percentage $\mathrm{vg}_{i}^{\text {tol }}$ that each generator admits to change its previously scheduled output. These constraints are established both for generators that were dispatched by the Market Operator (in set $S_{1}$ ) and generators having Bilateral Contracts (in set $S_{3}$ ). In case a generator bided in the day-ahead market and it was not scheduled (set $S_{2}$ ), its output can be adjusted by at most a percentage of its installed capacity, as indicated by constraint (10);

- constraints (11) indicate that the total adjustment of a generator is due to loss compensation and to variations required to enforce operation or security constraints. These total adjustments are bounded by the limits $\Delta \mathrm{Pg}_{i}^{\min }$ and $\Delta \mathrm{Pg}_{i}^{\max }$. These constraints are established for all generators in sets $S_{1}, S_{2}$ and $S_{3}$

- constraints (12) express the bounds on the load adjustments that were scheduled on the day-ahead market and for the ones having Bilateral Contracts, that is, for loads in sets $S_{1}$ and $S_{3}$; - regarding the capability diagram of synchronous generators, constraints (13) and (14) correspond to linearized expressions of curves 1 and 3 referred in Section 3.3 for generators that bided in the day-ahead market and for the ones having Bilateral Contracts. This means these constraints are written for all generators in sets $S_{1}, S_{2}$ and $S_{3}$. Apart from that, in these constraints $\mathrm{Pg}_{i}$ and $\mathrm{Qg}_{i}$ represent the values of the active and reactive power generations initially scheduled in the dayahead market or associated with the Bilateral Contracts and $\mathrm{Pg}_{i}^{\max }, \mathrm{Qg}_{i}^{\min }, \mathrm{Qg}_{i}^{\mathrm{a}}$ and $\mathrm{Qg}_{i}^{\mathrm{b}}$ are parameters that characterize the capability diagram of the generator $i$, according to Fig. 3; - constraints (15) impose the minimum and maximum bounds on the branch flows, expressed in terms of their apparent power. This power is written in terms of a linearized expression in function of voltage and phase variations;

- admitting that $\mathrm{Nb}$ represents the number of branches of the network, constraint (16) imposes that the sum of the loss deviations for all system branches is equal to the sum of the generator loss adjustment variables. This sum of adjustment variables is computed for all generators in sets $S_{1}, S_{2}$ and $S_{3}$. In the left side of this constraint, branch losses are given by linearized expressions in terms of voltage and phase variations;

- finally, constraints (17) and (18) represent linearized versions of the ac active and reactive injected power equations. For each node, the left hand side of these constraints corresponds to linearized expressions of the injected active and reactive power in function of voltage and phase variations. These linearized expressions are established using the first order terms of the Taylor Series for active and reactive injected powers. The right hand side of (17) includes the generation adjustment variables $\Delta \mathrm{Pg}_{i}^{\mathrm{L}}$ and $\Delta \mathrm{Pg}_{i}^{\mathrm{A}}$ and the load adjustment variables $\Delta \mathrm{Pd}_{i}^{\mathrm{A}}$ for all generators and loads connected to node $i$. In a similar way, the right hand side of (18) includes the reactive power generation and load adjustment variables for all generators and loads connected to node $i$.

Apart from minimizing active losses, operation security can also be improved if the system displays adequate reactive power margins. This aspect is not explicitly considered in this model but it is relevant since it is frequently contradictory regarding the minimization of active losses. However, it can be addressed if one changes some parameters in constraints (13) and (14) modeling the capability diagram of the generators. Regarding constraint (13), for instance, this would mean reducing the values of $\mathrm{Qg}_{i}^{\max }$ and $\mathrm{Qg}_{i}^{\mathrm{a}}$ in Fig. 3. This would create a margin between the upper curve of the capability diagram used by the System Operator and the real curve representing the rotor field current limit. This would correspond to a more constrained problem that could lead to an extra operation cost. This extra cost 
is explained because some generators could more easily reach their limit and therefore more costly generators would have to be used. Apart from that, transmission active losses would be larger because the generation active and reactive schedule leading to its minimization could be unfeasible given the more reduced reactive generation limits.

\subsection{Mathematical formulation-Model 2 (closed mechanism)}

The second model can be interpreted as an enhancement of Model 1 in terms of recognizing that there are two commercial subsystems responsible for relating generation and demand: the day-ahead market managed by the Market Operator and Bilateral Contracts. In view of these two mechanisms, Model 2 requires that each of these mechanisms is closed. This means that an adjustment on a generation or on a demand originally scheduled by the Market Operator must be balanced by an adjustment of a generation or by a demand also originally scheduled by the Market Operator. In a similar way, if a generator or a demand associated to a Bilateral Contract has to be changed, then the total generation associated to all Bilateral Contracts must be in equilibrium regarding the sum of the demands related to all Bilateral Contracts. The equilibrium of these two commercial mechanisms can be modeled by constraints (19) and (20) so that Model 2 includes the objective function (5) and constraints (6)-(20).

$$
\begin{aligned}
& \sum_{S_{1} \cup S_{2}} \Delta \mathrm{Pg}_{i}^{\mathrm{A}}=\sum_{S_{1}} \Delta \mathrm{Pd}_{j}^{\mathrm{A}} \\
& \sum_{S_{3}} \Delta \mathrm{Pg}_{i}^{\mathrm{A}}=\sum_{S_{3}} \Delta \mathrm{Pd}_{j}^{\mathrm{A}}
\end{aligned}
$$

Choosing between Model 1 and Model 2 is clearly a market design issue. However, it is clear that Model 2 is more constrained, and so the final value of the objective function obtained with Model 2 will be not inferior than the value of the objective function obtained with Model 1. Apart from that, it is possible that in some cases Model 2 does not have a solution given the topology of the network and the location of the generators and loads involved in each trading mechanism. Therefore, one may conceive a market design in which the System Operator runs in the first place Model 2. In case it has no solution, Model 1 could then be used given the increased flexibility it displays when not including constraints (19) and (20).

\section{Solution algorithm}

\subsection{Implementation of the SLP approach}

The optimization problems described in Section 3 were solved using the Sequential Linear Programming - SLP approach that is detailed in Fig. 4. According to this figure the original non-linear optimization problem is solved by successively linearizing it departing from the economic dispatch obtained by the Market Operator together with the information

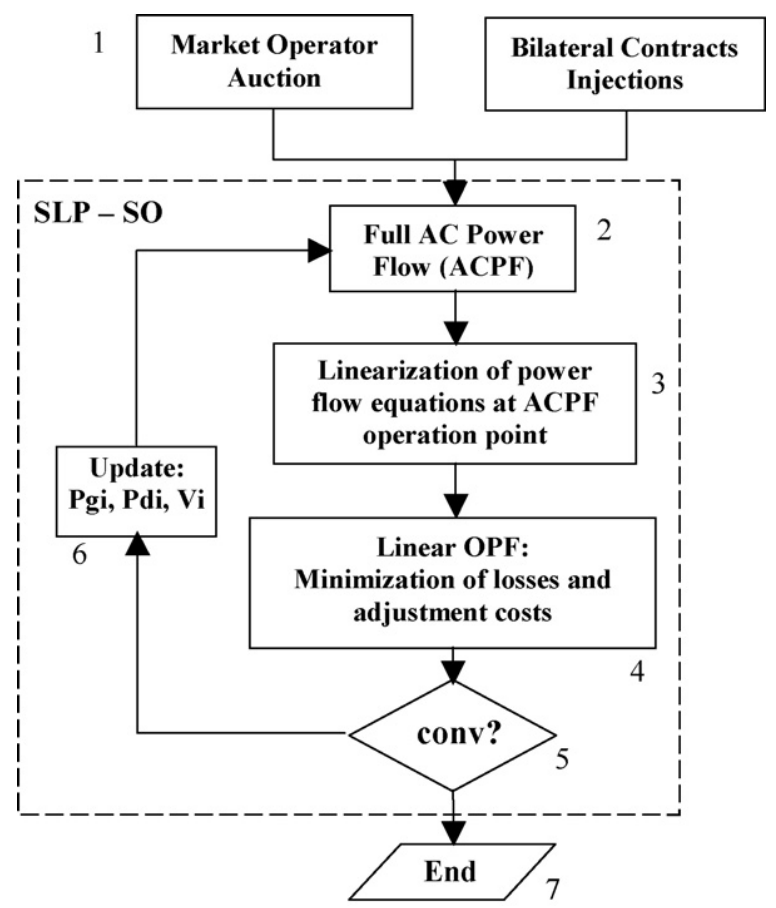

Fig. 4. Flowchart of the simplified SLP-based solution algorithm.

regarding the injected powers from Bilateral Contracts. According to Fig. 4, this solution algorithm is organized as follows:

- in Block 1, the Market Operator runs an uniform price bid based auction solving the linear problem (1)-(4) and using the simple bids detailed in Section 3.2;

- in Block 2, using this economic dispatch together with the injected powers associated with the Bilateral Contracts, the System Operator runs an ac power flow in order to characterize in a complete way this operation point of the system;

- in Block 3, the System Operator uses this operation point to linearize all non-linear expressions in the problem. This involves linearizing the branch active losses, the branch flow apparent power and the expressions of the active and reactive injected powers. This means getting linearized expressions to formulate constraints (15)-(18) detailed in Section 3.6. Apart from this linearization process, we also update several parameters of the formulation. For instance, the minimum and maximum voltage magnitude deviation limits in constraint (6) and the minimum and maximum apparent power flow deviation limits in constraint (15) are recomputed using the values obtained for these variables in the complete ac power flow study ran in Block 2 as well as the specified voltage ranges and branch apparent power limits;

- once this process is complete, the linearized problem is fully built. This means problem (5)-(18) for Model 1 or problem (5)-(20) for Model 2. The resulting optimization problem is solved in Block 4. It is important to refer that the absolute value of the deviations computed in each iteration is limited. This is important to guarantee that the new operation point resulting from solving this optimisation problem is not too far from the operation point used to linearize several expressions, as 
detailed before. If such maximum allowable changes were not constrained the iterative process could display an oscillatory behavior;

- in Block 5 one evaluates the convergence of the iterative process using the changes on voltages, phases and on the objective function in successive iterations. It should be noticed that the linearized optimization problem is formulated in terms of voltage and phase deviations. This means that the evaluation of the convergence from the point of view of voltage and phase changes is immediate since these deviations are directly available. When these deviations are smaller than specified tolerances, we get the solution for the non-linear problem;

- if convergence was not yet obtained, in Block 6 we update generations, loads, voltages and phases considering the adjustment values obtained from the linearized problem. This means we can run a new ac power flow to fully characterize the new operation point getting back to Block 2 .

\subsection{Computation of active/reactive nodal marginal prices}

Nodal marginal prices can be defined as the impact on the objective function of the problem due to a variation of the active or reactive demand in each node of the system. These prices typically display a geographical dispersion due to active losses as well as due to binding constraints, namely power flow branch limit constraints. These prices can be computed in a systematic way using the values of dual variables of the problem constraints. In this case, considering Model 1 and once the algorithm converges, the dual variables of the nodal active and reactive injected power Eqs. (17) and (18) are used to compute active and reactive nodal marginal prices. For Model 2 the dual variables of constraints (19) and (20) are also used to compute the corresponding active and reactive prices. In both cases, these prices reflect the impact on the objective function from changing the active or reactive load in each node.

\section{Case study}

\subsection{System data}

The models detailed in Section 3 and the solution approach in Section 4 were tested using a case study based on the IEEE 24 Bus Test System sketched in Fig. 5. The data of this system was originally presented in reference [20]. In our simulations we used 0.90 and 1.10 for the voltage limits and node 21 was the phase reference. Table 1 includes the data for Bilateral Contracts, namely the involved nodes, the load values and their adjustment prices. Table 2 completes this data including the points of the generator capability curve according to Fig. 3 and the generator adjustment bids considering the maximum adjustment percentage and the corresponding price. Table 3 contains the data for the day-ahead market generator bids including the structure of the bidding blocks corresponding to pairs (quantity, price). For these generators Table 4 contains the points of the capability curve and the adjustment bids including the maximum variation admitted by each generator and the adjustment price. Finally, Table 5 contains the active and reactive loads and the bidding

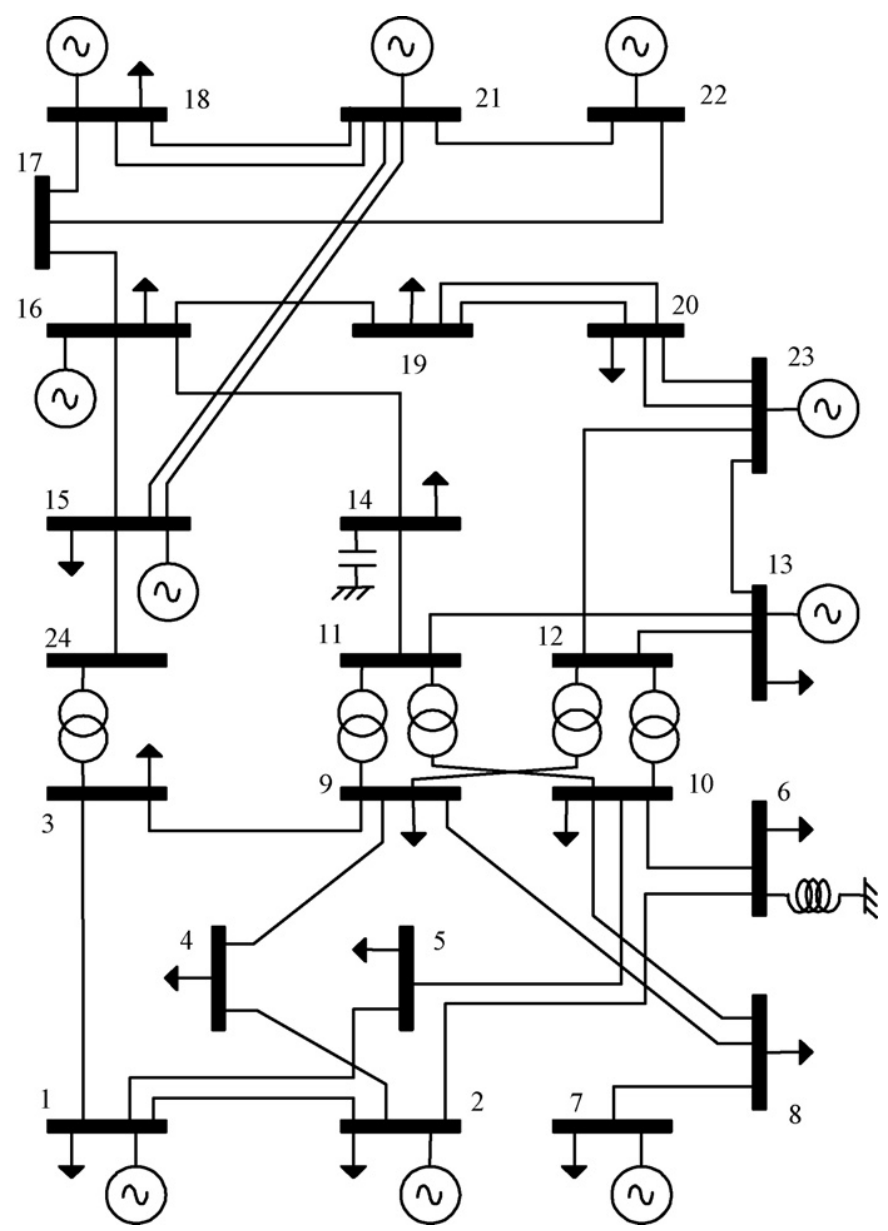

Fig. 5. IEEE 24 bus/38 branch Test System.

Table 1

Data for the Bilateral Contracts (generation and demand nodes, active and reactive load power and load adjustment price)

\begin{tabular}{|c|c|c|c|c|}
\hline $\begin{array}{l}\text { Generation } \\
\text { bus }\end{array}$ & $\begin{array}{l}\text { Demand } \\
\text { bus }\end{array}$ & $\mathrm{Pd}_{j}(\mathrm{MW})$ & $\mathrm{Qd}_{j}$ (Mvar) & $\mathrm{Cd}_{j}^{\mathrm{A}}(€ / \mathrm{MWh})$ \\
\hline \multirow[t]{3}{*}{7} & 2 & 20.00 & 4.06 & 288.0 \\
\hline & 5 & 22.00 & 5.00 & 296.0 \\
\hline & 14 & 10.00 & 2.92 & 289.0 \\
\hline \multirow[t]{3}{*}{13} & 7 & 30.00 & 6.09 & 290.0 \\
\hline & 13 & 18.00 & 3.00 & 292.0 \\
\hline & 18 & 13.00 & 3.00 & 286.0 \\
\hline \multirow[t]{2}{*}{15} & 1 & 10.00 & 1.43 & 299.0 \\
\hline & 9 & 16.00 & 2.28 & 280.0 \\
\hline \multirow[t]{3}{*}{16} & 10 & 16.00 & 2.28 & 294.0 \\
\hline & 13 & 15.00 & 2.00 & 292.0 \\
\hline & 20 & 20.00 & 4.00 & 286.0 \\
\hline \multirow[t]{2}{*}{18} & 18 & 25.00 & 4.72 & 276.0 \\
\hline & 19 & 35.00 & 4.99 & 299.0 \\
\hline \multirow[t]{3}{*}{21} & 5 & 25.00 & 6.52 & 296.0 \\
\hline & 16 & 10.00 & 1.43 & 287.0 \\
\hline & 20 & 24.00 & 4.94 & 286.0 \\
\hline \multirow[t]{3}{*}{22} & 4 & 15.00 & 2.14 & 285.0 \\
\hline & 5 & 25.00 & 6.52 & 296.0 \\
\hline & 13 & 12.00 & 1.41 & 292.0 \\
\hline
\end{tabular}


Table 2

Data for the capability curve and adjustment bids for generators having Bilateral Contracts

\begin{tabular}{|c|c|c|c|c|c|c|c|}
\hline \multirow[t]{2}{*}{ Bus $i$} & \multicolumn{5}{|c|}{ Points of the approximated capability curve } & \multicolumn{2}{|c|}{ Adjustment bids } \\
\hline & $\mathrm{Pg}_{i}^{\max }(\mathrm{MW})$ & $\mathrm{Qg}_{i}^{\max }$ (Mvar) & $\mathrm{Qg}_{i}^{\mathrm{a}}(\mathrm{Mvar})$ & $\mathrm{Qg}_{i}^{\mathrm{b}}$ (Mvar) & $\mathrm{Qg}_{i}^{\min }$ (Mvar) & $\mathrm{vg}_{i}^{\mathrm{tol}}(\%)$ & $\mathrm{Cg}_{i}^{\mathrm{A}}(€ / \mathrm{MWh})$ \\
\hline 7 & 100.0 & 65.0 & 50.0 & -40.0 & -60.0 & 40.0 & 108.0 \\
\hline 13 & 191.0 & 95.0 & 50.0 & -50.0 & -80.0 & 40.0 & 102.0 \\
\hline 15 & 115.0 & 70.0 & 40.0 & -25.0 & -50.0 & 40.0 & 111.0 \\
\hline 16 & 55.0 & 30.0 & 20.0 & -15.0 & -30.0 & 40.0 & 113.0 \\
\hline 18 & 100.0 & 65.0 & 30.0 & -20.0 & -60.0 & 40.0 & 101.0 \\
\hline 21 & 100.0 & 50.0 & 40.0 & -20.0 & -50.0 & 40.0 & 98.0 \\
\hline 22 & 80.0 & 55.0 & 40.0 & -25.0 & -40.0 & 40.0 & 107.0 \\
\hline
\end{tabular}

Table 3

Bidding blocks for the generators in the day-ahead market

\begin{tabular}{|c|c|c|c|c|c|c|}
\hline \multirow[t]{2}{*}{ Bus $i$} & \multicolumn{6}{|c|}{ Blocks of the generator selling bids } \\
\hline & $\mathrm{Pg}_{i}^{\text {bid } 1}(\mathrm{MW})$ & $\mathrm{Cg}_{i}^{1}(€ / \mathrm{MWh})$ & $\mathrm{Pg}_{i}^{\text {bid } 2}(\mathrm{MW})$ & $\mathrm{Cg}_{i}^{2}(€ / \mathrm{MWh})$ & $\mathrm{Pg}_{i}^{\text {bid } 3}(\mathrm{MW})$ & $\mathrm{Cg}_{i}^{3}(€ / \mathrm{MWh})$ \\
\hline 1 & 94.0 & 50.0 & 150.0 & 75.0 & 192.0 & 90.0 \\
\hline 2 & 96.0 & 60.0 & 154.0 & 80.0 & 192.0 & 90.0 \\
\hline 7 & 150.0 & 10.0 & 230.0 & 25.0 & 300.0 & 35.0 \\
\hline 13 & 300.0 & 15.0 & 450.0 & 24.0 & 591.0 & 35.0 \\
\hline 15 & 90.0 & 10.0 & 160.0 & 22.0 & 215.0 & 35.0 \\
\hline 16 & 100.0 & 15.0 & 155.0 & 35.0 & - & - \\
\hline 18 & 250.0 & 37.0 & 350.0 & 45.0 & 400.0 & 60.0 \\
\hline 21 & 250.0 & 35.0 & 350.0 & 44.0 & 400.0 & 60.0 \\
\hline 22 & 150.0 & 5.0 & 225.0 & 15.0 & 300.0 & 35.0 \\
\hline 23 & 300.0 & 15.0 & 550.0 & 24.0 & 660.0 & 35.0 \\
\hline
\end{tabular}

and adjustment prices for the loads that bided in the day-ahead market.

Using this data we conducted three simulations. In Cases 1 and 2 we used Model 1 described in Section 3.6. These two cases are different since in Case 1 there are no congested branches, while in Case 2 the limit of branch 7-8 was reduced to originate a congested situation. Finally, in Case 3 we used Model 2 for a congested situation similar to the one analyzed in Case 2.

\subsection{Results of Market Operator uniform price auction}

As referred before, the first phase of the solution algorithm corresponds to run the uniform price auction corresponding to the problem (1)-(4) using the data in Tables 3 and 5. The results of this Market Operator schedule are presented in Table 6 and the clearing price is $35.0 € / \mathrm{MWh}$.

\subsection{Case 1-System Operator (results using Model 1 without congestion)}

In Case 1, we ran the algorithm described in Section 4.1 using Model 1. The optimization problem corresponding to this model was formulated using the results of the Market Operator dispatch in Table 6 and the Bilateral Contract injections in Table 1. As a result we obtained the final solution presented in Table 7 . This Table includes the voltages and phases and the final dispatch values for the generators having Bilateral Contracts and for the ones that bided in the day-ahead market. For both of them, the

Table 4

Data for the capability curve and adjustment bids for the generators in the day-ahead market

\begin{tabular}{|c|c|c|c|c|c|c|c|}
\hline \multirow[t]{2}{*}{ Bus $i$} & \multicolumn{5}{|c|}{ Points of the approximated capability curve } & \multicolumn{2}{|c|}{ Adjustment bids } \\
\hline & $\mathrm{Pg}_{i}^{\max }(\mathrm{MW})$ & $\mathrm{Qg}_{i}^{\max }$ (Mvar) & $\mathrm{Qg}_{i}^{\mathrm{a}}$ (Mvar) & $\mathrm{Qg}_{i}^{\mathrm{b}}$ (Mvar) & $\mathrm{Qg}_{i}^{\min }$ (Mvar) & $\mathrm{vg}_{i}^{\mathrm{tol}}(\%)$ & $\mathrm{Cg}_{i}^{\mathrm{A}}(€ / \mathrm{MWh})$ \\
\hline 1 & 192.0 & 130.0 & 90.0 & -70.0 & -100.0 & 40.0 & 110.0 \\
\hline 2 & 192.0 & 120.0 & 80.0 & -60.0 & -90.0 & 40.0 & 115.0 \\
\hline 7 & 300.0 & 200.0 & 150.0 & -160.0 & -180.0 & 40.0 & 120.0 \\
\hline 13 & 591.0 & 250.0 & 150.0 & -150.0 & -200.0 & 40.0 & 105.0 \\
\hline 15 & 215.0 & 150.0 & 120.0 & -85.0 & -100.0 & 40.0 & 100.0 \\
\hline 16 & 155.0 & 100.0 & 70.0 & -55.0 & -80.0 & 40.0 & 112.0 \\
\hline 18 & 400.0 & 250.0 & 150.0 & -120.0 & -200.0 & 40.0 & 130.0 \\
\hline 21 & 400.0 & 250.0 & 150.0 & -100.0 & -200.0 & 40.0 & 160.0 \\
\hline 22 & 300.0 & 220.0 & 125.0 & -100.0 & -180.0 & 40.0 & 103.0 \\
\hline 23 & 660.0 & 300.0 & 185.0 & -165.0 & -280.0 & 40.0 & 118.0 \\
\hline
\end{tabular}


Table 5

Data for the demand bids in the day-ahead market

\begin{tabular}{cclcl}
\hline Bus $j$ & $\mathrm{Pd}_{j}^{\mathrm{bid}}(\mathrm{MW})$ & $\mathrm{Qd}_{j}(\mathrm{Mvar})$ & $\mathrm{Cd}_{j}(€ / \mathrm{MWh})$ & $\mathrm{Cd}_{j}^{\mathrm{A}}(€ / \mathrm{MWh})$ \\
\hline 1 & 108.0 & 27.1 & 80.0 & 295.0 \\
2 & 97.0 & 24.3 & 93.0 & 290.0 \\
3 & 180.0 & 36.6 & 99.0 & 289.0 \\
4 & 74.0 & 18.5 & 88.0 & 288.0 \\
5 & 71.0 & 14.4 & 101.0 & 296.0 \\
6 & 136.0 & 34.1 & 50.0 & 300.0 \\
7 & 125.0 & 25.4 & 91.0 & 285.0 \\
8 & 171.0 & 24.4 & 85.0 & 295.0 \\
9 & 175.0 & 35.5 & 75.0 & 296.0 \\
10 & 195.0 & 48.9 & 35.0 & 288.0 \\
13 & 265.0 & 53.8 & 20.0 & 287.0 \\
14 & 194.0 & 48.6 & 92.0 & 305.0 \\
15 & 317.0 & 64.4 & 94.0 & 301.0 \\
16 & 100.0 & 25.1 & 92.0 & 294.0 \\
18 & 333.0 & 67.6 & 90.0 & 296.0 \\
19 & 181.0 & 36.8 & 36.0 & 298.0 \\
20 & 128.0 & 32.1 & 96.0 & 291.0 \\
\hline
\end{tabular}

generator adjustments are decoupled in terms of the changes required to balance active losses, $\Delta \mathrm{Pg}_{i}^{\mathrm{L}}$, and of the adjustments needed to enforce violated constraints, $\Delta \mathrm{Pg}_{i}^{\mathrm{A}}$, namely voltage and branch flow limits. In this case, the results indicate that the adjustments are only due to the compensation of active losses and that this compensation is done in generators 1 and 2 with the values of 6.35 and 58.35 MW. Since the Market Operator did not initially dispatch these two generators, their final outputs coincide with the adjustments required to balance active losses. Regarding the demand, it is important to notice that load adjustment bids were not used. Therefore, the final dispatched loads are the same regarding the ones cleared by the Market Operator and the ones in Table 1 for the Bilateral Contracts.

As referred before, active losses are balanced in nodes 1 and 2, that is, in two generators that bided in the day-ahead market but that were not scheduled. This occurs although the formulation discriminates between Market Operator and Bilateral Contract generators and for each class there are adjustment variables related with the compensation of active losses and with the solution of system constraints. The final values of these adjustment variables will certainly depend on the location of the generators, on their initial dispatched quantities, on their limits and on the adjustment prices. In this simulation, active losses are allocated

Table 6

Results of the Market Operator dispatch

\begin{tabular}{cccccc}
\hline Bus $i$ & $\mathrm{Pd}_{i}(\mathrm{MW})$ & $\mathrm{Pg}_{i}(\mathrm{MW})$ & $\mathrm{Bus} i$ & $\mathrm{Pd}_{i}(\mathrm{MW})$ & $\mathrm{Pg}_{i}(\mathrm{MW})$ \\
\hline 1 & 108.0 & 0.0 & 13 & 0.0 & 579.62 \\
2 & 97.0 & 0.0 & 14 & 194.0 & - \\
3 & 180.0 & - & 15 & 317.0 & 206.76 \\
4 & 74.0 & - & 16 & 100.0 & 146.76 \\
5 & 71.0 & - & 18 & 333.0 & 0.0 \\
6 & 136.0 & - & 19 & 181.0 & - \\
7 & 125.0 & 290.89 & 20 & 128.0 & - \\
8 & 171.0 & - & 21 & - & 238.03 \\
9 & 175.0 & - & 22 & - & 290.64 \\
10 & 12.01 & - & 23 & - & 649.31 \\
\hline
\end{tabular}

to generators in nodes 1 and 2 because this corresponds to the strategy that minimizes them. It should be realized that the system is quite unbalanced from the point of view of the dispatched generators. In fact, generators connected to nodes 1 and 2 were not dispatched by the Market Operator (see the results in Table 6) due to their high bid prices (see data in Table 3). This means that almost all generation is concentrated in nodes 13, 15, 16, 21, 22 and 23. As a result, the power tends to flow from these nodes located in the upper part of the system in Fig. 5 to its lower part. Increasing the generation in nodes 13, 15, 16, 21, 22 and 23 to balance active losses would increase even more this unbalance not contributing to minimize active losses. On the contrary, if active losses are balanced in nodes 1 and 2, generation will be better distributed along the system thus contributing to minimize active losses. This explains why active losses are allocated to these two nodes, in fact to two Market Operator generators.

Finally, it should be noticed that if Model 2 was used for this case the results would be the same. In fact, the initial Market Operator and Bilateral Contract schedules are balanced and they remain like that because it was not necessary to use the adjustment generation or load bids. This means that the integration of constraints (19) and (20) included in Model 2 would not change the final solution presented in Table 7 for Case 1.

\subsection{Case 2-System Operator (results using Model 1 with congestion)}

In a second step, the limit of branch 7-8 was reduced to 150 MVA leading to Case 2. We ran again the SLP algorithm using Model 1 and Table 8 presents the final results for voltages, phases as well as the dispatch values. Due to the congestion in branch 7-8, there are several generators that increase or decrease their output. Regarding the Market Operator generators, the output of generator 7 is reduced by $17.09 \mathrm{MW}$ and the output of generator 15 is increased by 8.24 MW. Regarding the generators having Bilateral Contracts, generator 7 decreases its output by $20.80 \mathrm{MW}$ while the outputs of generators 18 and 21 are increased by 6.06 and $23.60 \mathrm{MW}$. As a whole, this means that generator increases and reductions are globally balanced but since Model 1 does not consider constraints (19) and (20) there is a net transference of $8.86 \mathrm{MW}$ from the Market Operator cleared quantity to the Bilateral Contracts.

For the same reason referred in Section 5.3, branch active losses are balanced by generators in nodes 1 and 2, 26.12 and 40.83 MW. Therefore, these generators move from a situation in which they were not dispatched by the Market Operator to a final output of 26.12 and $40.83 \mathrm{MW}$ respectively. Finally, it should be referred that the demand adjustment bids were not used.

\subsection{Case 3-System Operator (results using Model 2 with congestion)}

We then used Model 2 in a congested situation similar to one described in Case 2. For this situation, Table 9 presents the final values obtained for voltages, phases and for the dispatch. The congestion in branch 7-8 determines variations of generator outputs cleared by the Market Operator and having 
Table 7

Case 1-final dispatch of the System Operator

\begin{tabular}{|c|c|c|c|c|c|c|c|c|c|c|c|c|c|c|}
\hline \multirow[t]{2}{*}{ Bus $i$} & \multirow[t]{2}{*}{$\mathrm{Vi}(\mathrm{pu})$} & \multirow[t]{2}{*}{$\theta_{i}\left({ }^{\circ}\right)$} & \multicolumn{6}{|c|}{ Market Operator generators and loads } & \multicolumn{6}{|c|}{ Generators and loads having Bilateral Contracts } \\
\hline & & & $\Delta \mathrm{Pg}_{i}^{\mathrm{A}}(\mathrm{MW})$ & $\Delta \mathrm{Pg}_{i}^{\mathrm{L}}(\mathrm{MW})$ & $\mathrm{Pg}_{i}(\mathrm{MW})$ & $\mathrm{Qg}_{i}$ (Mvar) & $\mathrm{Pd}_{i}(\mathrm{MW})$ & $\mathrm{Qd}_{i}(\mathrm{Mvar})$ & $\Delta \mathrm{Pg}_{i}^{\mathrm{A}}(\mathrm{MW})$ & $\Delta \mathrm{Pg}_{i}^{\mathrm{L}}(\mathrm{MW})$ & $\mathrm{Pg}_{i}(\mathrm{MW})$ & $\mathrm{Qg}_{i}(\mathrm{Mvar})$ & $\mathrm{Pd}_{i}(\mathrm{MW})$ & $\mathrm{Qd}_{i}(\mathrm{Mvar})$ \\
\hline 1 & 1.02 & -24.75 & 0.00 & 6.35 & 6.35 & 106.83 & 108.00 & 27.07 & - & - & - & - & 10.00 & 1.43 \\
\hline 2 & 1.02 & -24.61 & 0.00 & 58.35 & 58.35 & 107.84 & 97.00 & 24.31 & - & - & - & - & 20.00 & 4.06 \\
\hline 3 & 1.01 & -16.52 & - & - & - & - & 180.00 & 36.55 & - & - & - & - & - & - \\
\hline 4 & 0.99 & -21.02 & - & - & - & - & 74.00 & 18.55 & - & - & - & - & 15.00 & 2.14 \\
\hline 5 & 0.98 & -22.59 & - & - & - & - & 71.00 & 14.42 & - & - & - & - & 72.00 & 18.05 \\
\hline 6 & 0.98 & -19.50 & - & - & - & - & 136.00 & 34.09 & - & - & - & - & - & - \\
\hline 7 & 1.10 & -7.60 & 0.00 & 0.00 & 290.89 & 81.99 & 125.00 & 25.38 & 0.00 & 0.00 & 52.00 & 18.03 & 30.00 & 6.09 \\
\hline 8 & 1.04 & -12.87 & - & - & - & - & 171.00 & 24.37 & - & - & - & - & - & - \\
\hline 9 & 1.02 & -13.12 & - & - & - & - & 175.00 & 35.54 & - & - & - & - & 16.00 & 2.28 \\
\hline 10 & 1.01 & -13.43 & - & - & - & - & 12.01 & 3.01 & - & - & - & - & 16.00 & 2.28 \\
\hline 11 & 1.04 & -5.68 & - & - & - & - & - & - & - & - & - & - & - & - \\
\hline 12 & 1.04 & -3.72 & - & - & - & - & - & - & - & - & - & - & - & - \\
\hline 13 & 1.10 & 2.36 & 0.00 & 0.00 & 579.623 & 138.64 & 0.00 & 0.00 & 0.00 & 0.00 & 61.00 & 65.58 & 45.00 & 6.41 \\
\hline 14 & 1.05 & -5.98 & - & - & - & - & 194.00 & 48.62 & - & - & - & - & 10.00 & 2.92 \\
\hline 15 & 1.09 & -2.63 & 0.00 & 0.00 & 206.76 & 121.15 & 317.00 & 64.37 & 0.00 & 0.00 & 26.00 & 63.22 & - & - \\
\hline 16 & 1.09 & -2.38 & 0.00 & 0.00 & 146.76 & 71.60 & 100.00 & 25.06 & 0.00 & 0.00 & 51.00 & 20.73 & 10.00 & 1.43 \\
\hline 17 & 1.10 & -1.23 & - & - & - & - & - & - & - & - & - & - & - & - \\
\hline 18 & 1.10 & -1.62 & 0.00 & 0.00 & 0.00 & 141.14 & 333.00 & 67.62 & 0.00 & 0.00 & 60.00 & 14.40 & 38.00 & 7.72 \\
\hline 19 & 1.08 & -1.53 & - & - & - & - & 181.00 & 36.75 & - & - & - & - & 35.00 & 4.99 \\
\hline 20 & 1.09 & 1.23 & - & - & - & - & 128.00 & 32.08 & - & - & - & - & 44.00 & 8.94 \\
\hline 21 & 1.10 & 0.00 & 0.00 & 0.00 & 238.03 & 21.34 & - & - & 0.00 & 0.00 & 59.00 & 3.92 & - & - \\
\hline 22 & 1.10 & 6.28 & 0.00 & 0.00 & 290.64 & -22.57 & - & - & 0.00 & 0.00 & 52.00 & 4.17 & - & - \\
\hline 23 & 1.10 & 3.58 & 0.00 & 0.00 & 649.31 & 128.74 & - & - & - & - & - & - & - & - \\
\hline 24 & 1.05 & -7.56 & - & - & - & - & - & - & - & - & - & - & - & - \\
\hline
\end{tabular}


Table 8

Case 2-final dispatch of the System Operator

\begin{tabular}{|c|c|c|c|c|c|c|c|c|c|c|c|c|c|c|}
\hline \multirow[t]{2}{*}{ Bus $i$} & \multirow[t]{2}{*}{$\mathrm{Vi}(\mathrm{pu})$} & \multirow[t]{2}{*}{$\theta_{i}\left({ }^{\circ}\right)$} & \multicolumn{6}{|c|}{ Market Operator generators and loads } & \multicolumn{6}{|c|}{ Generators and loads having Bilateral Contracts } \\
\hline & & & $\Delta \mathrm{Pg}_{i}^{\mathrm{A}}(\mathrm{MW})$ & $\Delta \mathrm{Pg}_{i}^{\mathrm{L}}(\mathrm{MW})$ & $\mathrm{Pg}_{i}(\mathrm{MW})$ & $\mathrm{Qg}_{i}$ (Mvar) & $\mathrm{Pd}_{i}(\mathrm{MW})$ & $\mathrm{Qd}_{i}(\mathrm{Mvar})$ & $\Delta \mathrm{Pg}_{i}^{\mathrm{A}}(\mathrm{MW})$ & $\Delta \mathrm{Pg}_{i}^{\mathrm{L}}(\mathrm{MW})$ & $\mathrm{Pg}_{i}(\mathrm{MW})$ & $\mathrm{Qg}_{i}$ (Mvar) & $\mathrm{Pd}_{i}(\mathrm{MW})$ & $\mathrm{Qd}_{i}$ (Mvar) \\
\hline 1 & 1.01 & -26.56 & 0.00 & 26.12 & 26.12 & 124.56 & 108.00 & 27.07 & - & - & - & - & 10.00 & 1.43 \\
\hline 2 & 1.01 & -26.57 & 0.00 & 40.83 & 40.83 & 111.49 & 97.00 & 24.31 & - & - & - & - & 20.00 & 4.06 \\
\hline 3 & 1.00 & -17.70 & - & - & - & - & 180.00 & 36.55 & - & - & - & - & - & - \\
\hline 4 & 0.97 & -22.85 & - & - & - & - & 74.00 & 18.55 & - & - & - & - & 15.00 & 2.14 \\
\hline 5 & 0.96 & -24.38 & - & - & - & - & 71.00 & 14.42 & - & - & - & - & 72.00 & 18.05 \\
\hline 6 & 0.96 & -21.24 & - & - & - & - & 136.00 & 34.09 & - & - & - & - & - & - \\
\hline 7 & 0.98 & -10.08 & -17.09 & 0.00 & 273.80 & 24.62 & 125.00 & 25.38 & -20.80 & 0.00 & 31.20 & 7.94 & 30.00 & 6.09 \\
\hline 8 & 0.96 & -15.72 & - & - & - & - & 171.00 & 24.37 & - & - & - & - & - & - \\
\hline 9 & 1.00 & -14.59 & - & - & - & - & 175.00 & 35.54 & - & - & - & - & 16.00 & 2.28 \\
\hline 10 & 0.99 & -14.87 & - & - & - & - & 12.01 & 3.01 & - & - & - & - & 16.00 & 2.28 \\
\hline 11 & 1.03 & -6.51 & - & - & - & - & - & - & - & - & - & - & - & - \\
\hline 12 & 1.03 & -4.60 & - & - & - & - & - & - & - & - & - & - & - & - \\
\hline 13 & 1.10 & 1.60 & 0.00 & 0.00 & 579.63 & 151.93 & 0.00 & 0.00 & 0.00 & 0.00 & 61.00 & 80.63 & 45.00 & 6.41 \\
\hline 14 & 1.04 & -6.52 & - & - & - & - & 194.00 & 48.62 & - & - & - & - & 10.00 & 2.92 \\
\hline 15 & 1.09 & -2.82 & 8.24 & 0.00 & 215.00 & 120.00 & 317.00 & 64.37 & 0.00 & 0.00 & 26.00 & 63.22 & - & - \\
\hline 16 & 1.09 & -2.62 & 0.00 & 0.00 & 146.76 & 71.60 & 100.00 & 25.06 & 0.00 & 0.00 & 51.00 & 20.73 & 10.00 & 1.43 \\
\hline 17 & 1.10 & -1.33 & - & - & - & - & - & - & - & - & - & - & - & - \\
\hline 18 & 1.10 & -1.65 & 0.00 & 0.00 & 0.00 & 147.96 & 333.00 & 67.62 & 6.06 & 0.00 & 66.06 & 11.63 & 38.00 & 7.72 \\
\hline 19 & 1.08 & -1.91 & - & - & - & - & 181.00 & 36.75 & - & - & - & - & 35.00 & 4.99 \\
\hline 20 & 1.09 & 0.73 & - & - & - & - & 128.00 & 32.08 & - & - & - & - & 44.00 & 8.94 \\
\hline 21 & 1.10 & 0.00 & 0.00 & 0.00 & 238.03 & 35.14 & - & - & 23.60 & 0.00 & 82.60 & 6.79 & - & - \\
\hline 22 & 1.10 & 6.25 & 0.00 & 0.00 & 290.64 & -24.48 & - & - & 0.00 & 0.00 & 52.00 & 4.32 & - & - \\
\hline 23 & 1.10 & 3.00 & 0.00 & 0.00 & 649.31 & 163.01 & - & - & - & - & - & - & - & - \\
\hline 24 & 1.04 & -8.04 & - & - & - & - & - & - & - & - & - & - & - & - \\
\hline
\end{tabular}


Table 9

Case 3-final dispatch of the System Operator

\begin{tabular}{|c|c|c|c|c|c|c|c|c|c|c|c|c|c|c|}
\hline \multirow[t]{2}{*}{ Bus $i$} & \multirow[t]{2}{*}{$\mathrm{Vi}(\mathrm{pu})$} & \multirow[t]{2}{*}{$\theta_{i}\left({ }^{\circ}\right)$} & \multicolumn{6}{|c|}{ Market Operator generators and loads } & \multicolumn{6}{|c|}{ Generators and loads having Bilateral Contracts } \\
\hline & & & $\Delta \mathrm{Pg}_{i}^{\mathrm{A}}(\mathrm{MW})$ & $\Delta \mathrm{Pg}_{i}^{\mathrm{L}}(\mathrm{MW})$ & $\operatorname{Pg}_{i}(\mathrm{MW})$ & $\mathrm{Qg}_{i}$ (Mvar) & $\mathrm{Pd}_{i}(\mathrm{MW})$ & $\mathrm{Qd}_{i}(\mathrm{Mvar})$ & $\Delta \mathrm{Pg}_{i}^{\mathrm{A}}(\mathrm{MW})$ & $\Delta \mathrm{Pg}_{i}^{\mathrm{L}}(\mathrm{MW})$ & $\mathrm{Pg}_{i}(\mathrm{MW})$ & $\mathrm{Qg}_{i}$ (Mvar) & $\mathrm{Pd}_{i}(\mathrm{MW})$ & $\mathrm{Qd}_{i}(\mathrm{Mvar})$ \\
\hline 1 & 1.01 & -26.31 & 2.81 & 25.62 & 28.43 & 124.08 & 108.00 & 27.07 & - & - & - & - & 10.00 & 1.43 \\
\hline 2 & 1.01 & -26.32 & 0.00 & 41.00 & 41.00 & 111.46 & 97.00 & 24.31 & - & - & - & - & 20.00 & 4.06 \\
\hline 3 & 1.00 & -17.58 & - & - & - & - & 180.00 & 36.55 & - & - & - & - & - & - \\
\hline 4 & 0.97 & -22.67 & - & - & - & - & 74.00 & 18.55 & - & - & - & - & 15.00 & 2.14 \\
\hline 5 & 0.97 & -24.18 & - & - & - & - & 71.00 & 14.42 & - & - & - & - & 72.00 & 18.05 \\
\hline 6 & 0.96 & -21.07 & - & - & - & - & 136.00 & 34.09 & - & - & - & - & - & - \\
\hline 7 & 0.98 & -9.97 & -17.09 & 0.00 & 273.79 & 24.15 & 125.00 & 25.38 & -20.80 & 0.00 & 31.20 & 8.19 & 30.00 & 6.09 \\
\hline 8 & 0.96 & -15.60 & - & - & - & - & 171.00 & 24.37 & - & - & - & - & - & - \\
\hline 9 & 1.00 & -14.48 & - & - & - & - & 175.00 & 35.54 & - & - & - & - & 16.00 & 2.28 \\
\hline 10 & 0.99 & -14.74 & - & - & - & - & 12.01 & 3.01 & - & - & - & - & 16.00 & 2.28 \\
\hline 11 & 1.03 & -6.45 & - & - & - & - & - & - & - & - & - & - & - & - \\
\hline 12 & 1.03 & -4.53 & - & - & - & - & - & - & - & - & - & - & - & - \\
\hline 13 & 1.10 & 1.64 & 0.00 & 0.00 & 579.63 & 151.93 & 0.00 & 0.00 & 0.00 & 0.00 & 61.00 & 80.63 & 45.00 & 6.42 \\
\hline 14 & 1.04 & -6.47 & - & - & - & - & 194.00 & 48.62 & - & - & - & - & 10.00 & 2.92 \\
\hline 15 & 1.09 & -2.80 & 8.24 & 0.00 & 215.00 & 120.00 & 317.00 & 64.37 & 0.00 & 0.00 & 26.00 & 63.22 & - & - \\
\hline 16 & 1.09 & -2.60 & 0.00 & 0.00 & 146.76 & 71.60 & 100.00 & 25.06 & 0.00 & 0.00 & 51.00 & 20.73 & 10.00 & 1.43 \\
\hline 17 & 1.10 & -1.32 & - & - & - & - & - & - & - & - & - & - & - & - \\
\hline 18 & 1.10 & -1.66 & 0.00 & 0.00 & 0.00 & 148.98 & 333.00 & 67.62 & 0.00 & 0.00 & 60.00 & 13.60 & 38.00 & 7.72 \\
\hline 19 & 1.08 & -1.88 & - & - & - & - & 181.00 & 36.75 & - & - & - & - & 35.00 & 4.99 \\
\hline 20 & 1.09 & 0.76 & - & - & - & - & 128.00 & 32.08 & - & - & - & - & 44.00 & 8.94 \\
\hline 21 & 1.10 & 0.00 & 0.00 & 0.00 & 238.03 & 36.84 & - & - & 20.80 & 0.00 & 79.80 & 6.50 & - & - \\
\hline 22 & 1.10 & 6.41 & 6.05 & 0.00 & 296.68 & -30.27 & - & - & 0.00 & 0.00 & 52.00 & 2.92 & - & - \\
\hline 23 & 1.10 & 3.03 & 0.00 & 0.00 & 649.31 & 164.14 & - & - & - & - & - & - & - & - \\
\hline 24 & 1.04 & -8.00 & - & - & - & - & - & - & - & - & - & - & - & - \\
\hline
\end{tabular}




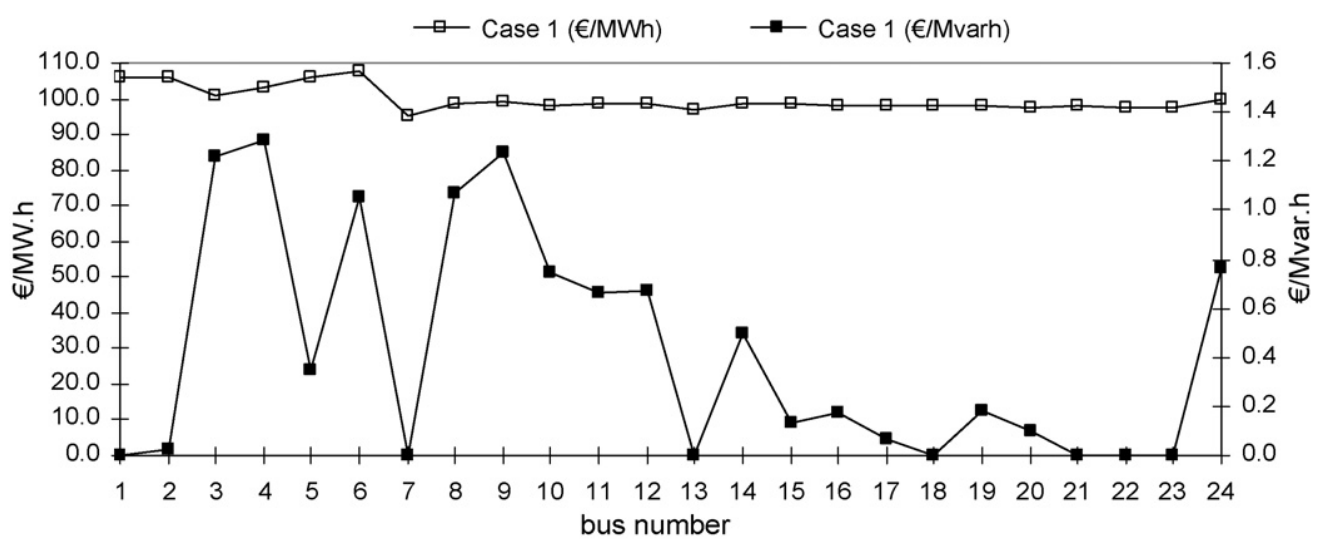

Fig. 6. Profiles of the active and reactive nodal marginal prices for Case 1 .

Bilateral Contracts. Besides, constraints (19) and (20) impose that each contractual subsystem is kept balanced. As a result, for generators cleared by the Market Operator, generators 1, 15 and 22 increase their output by $2.81,8.24$ and $6.05 \mathrm{MW}$, while generator 7 reduces its output by $17.09 \mathrm{MW}$ thus leading to a balanced situation, according to constraint (19). Regarding the generators having Bilateral Contracts, generator 7 decreases its output by $20.80 \mathrm{MW}$ and generator 21 increases it by the same amount also leading to a balanced situation in line with constraint (20).

Branch active losses are once again balanced by the generators in nodes 1 and 2, 25.62 and $41.00 \mathrm{MW}$. This means that considering both the adjustments to enforce operation constraints and the adjustments required to balance losses, the final outputs of generators 1 and 2 are 28.43 and $41.00 \mathrm{MW}$ since they were originally not dispatch by the Market Operator, as indicated in Table 6.

\subsection{Nodal marginal active and reactive prices}

Figs. 6 and 7 display the profiles of the active and reactive nodal marginal prices obtained for Cases 1 and 3. Regarding Case 1, Fig. 6 indicates that the active power nodal marginal prices are very uniform. The active power marginal price in node $k$ reflects the impact of increasing the active load in that node. This increase has two consequences given that a gen- erator must balance it and there is also an impact on branch losses. In Case 1 most of the nodal marginal prices have values close to $98.0 € / \mathrm{MWh}$ since this is the cheapest generator adjustment bid, in this case in node 21 . However, for nodes 1-6 the nodal prices are higher indicating that if the load increase occurs in one of these nodes this change is balanced by the adjustment offer of generator 1 that has an adjustment price of $110.0 € / \mathrm{MWh}$.

Regarding the reactive nodal marginal prices they are much more reduced than the active power prices confirming the idea initially referred in Sections 1 and 2 and they basically reflect the impact on branch losses from increasing the load in each node. It is interesting to notice that the reactive power marginal price in nodes $1,7,13,18,21,22$ and 23 is zero since the operation points of these generators are not over their capability curve. This means that these generators can increase their reactive output without having to reduce their active generation. Therefore, the generators in these nodes directly balance at zero cost a reactive load increase in any of them. However, if there is an increase of the reactive load in node 2 the reactive power price is not zero. The generator connected to node 2 is at the limit given its capability diagram and so another generator must balance a reactive load increase in node 2 with the corresponding impact in active branch losses. The same reason explains the non-zero reactive prices obtained for nodes 15 and 16 although there are generators connected to these nodes. For nodes not having gen-

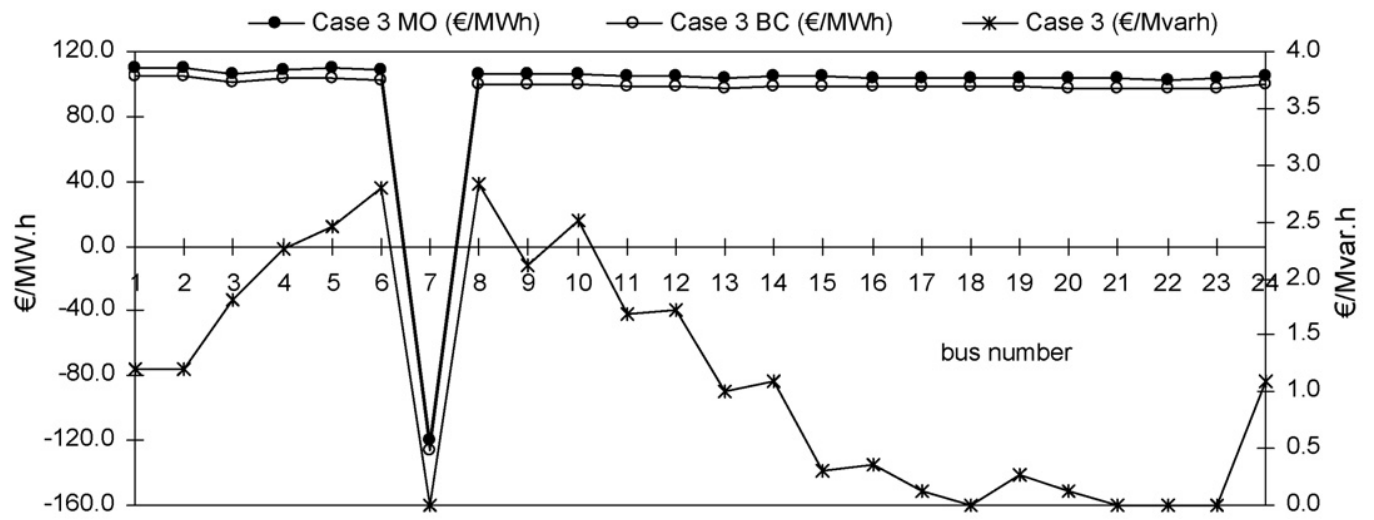

Fig. 7. Profiles of the active and reactive nodal marginal prices for Case 3. 
erators connected to them it is clear that the reactive marginal prices are above zero since a reactive power increase has to be balanced in a different node leading to an impact on active losses.

Regarding the nodal marginal prices obtained for Case 3, Fig. 7 displays two curves for active prices, one of them for the Market Operator subsystem and the second for the Bilateral Contracts. This difference comes from the fact that Model 2 includes two additional equality constraints when compared with Model 1. Accordingly, if one increases the active demand in node $k$ related with the Market Operator subsystem the corresponding marginal price results from the dual variable of the injected active power constraint (17) for node $k$ plus the dual variable of constraint (19). If one considers a demand related to a Bilateral Contract, the active power marginal price in node $k$ is obtained using the dual variable of the injected active power constraint (17) for node $k$ plus the dual variable of constraint (20).

In Fig. 7 and except for the price in node 7 , the active power prices obtained for the Market Operator subsystem are very homogeneous and close to $103 € / \mathrm{MWh}$. This value corresponds to the adjustment price offered by the generator in node 22. This is the cheapest adjustment price (see the last column of Table 4) and the operation point of this generator (296.68 MW and -30.27 Mvar as indicated in Table 9) is not on the limit. The prices obtained for nodes 11 and 12, for instance, are 105.07 €/MWh and 105.06€/MWh which are above the adjustment price offered by the generator in node 13 $(105 € / \mathrm{MWh})$. However, the operation point of the generator in node 13 (597.63 MW and 151.93 Mvar as indicated in Table 9) is already over the capability curve of this generator turning it impossible to use it for this purpose.

Regarding the Bilateral Contract subsystem, and once again except for node 7 , the active power prices are close to $98 € / \mathrm{MWh}$ and this value coincides with the adjustment price of the generator in node 21 (see data in Table 2). It should be realized that this is the cheapest adjustment price for Bilateral Contract generators and that the operation point of the generator in node 21 (79.80 MW and 6.50 Mvar as indicated in Table 9) is well below the upper curve of its capability diagram.

The negative prices obtained for the active power in node 7 deserve an explanation. The Market Operator originally assigned 290.89 MW to generator 7, as indicated in Table 6. When running Case 3 and using 150 MVA for the flow limit in branch 7-8, this generator has an adjustment of $-17.09 \mathrm{MW}$ so that its output is reduced to $273.79 \mathrm{MW}$. If the load in node 7 increases by 1 unit, then generator 7 can generate $274.79 \mathrm{MW}$ and this extragenerated MW directly supplies the extra load MW. This means that this generator should now be adjusted only by $-16.09 \mathrm{MW}$ instead of $-17.09 \mathrm{MW}$. Since the adjustment price of generator 7 is $120 € / M W h$ for the Market Operator subsystem (see data in Table 4), this reduction of 1 unit in its adjustment represents a reduction of $120 € / \mathrm{MWh}$ in the cost function of the problem. This negative impact on the cost function from increasing the load in node 7 explains the negative marginal price obtained for this node. A similar analysis explains the negative price obtained for node 7 for the Bilateral Contract subsystem.
Regarding the reactive power prices they display a larger variation when compared to the ones in Fig. 6. The prices in nodes 7, $18,21,22$ and 23 remain zero for the same reason already indicated for Case 1 . The prices in other generation nodes, namely in node 1 , are not zero since the operation point of the generator connected to node 1 (28.43 MW and 124.08 Mvar as indicated in Table 9) is on the limit giving the corresponding capability diagram. This means that a reactive load increase in node 1 has to be balanced in another bus leading to an impact on active losses and therefore on the objective function of the problem. For the remaining nodes, the reactive power prices are generally higher than in Case 1 since the impact on active losses due to an increase on the reactive power in those nodes is also larger. This larger impact can be easily checked if one runs power flow studies based on the final operation points obtained for Cases 1 and 3 and considering then small changes on reactive power values in several nodes.

\subsection{Implementation and performance of the SLP approach}

When implementing the SLP approach we restrained the maximum allowable changes in the variables of the linearized problem as referred in Section 4.1. For the 24 Bus Test System we used a maximum allowable change of $0.001 \mathrm{pu}$. It should be realized that this value represents a compromise between the computational efficiency of the algorithm and the need to prevent oscillations in the iterative process. Regarding the three cases described in Sections 5.3, 5.4 and 5.5 the number of iterations required to get convergence was 25,57 and 64 and the corresponding CPU time was 5.6, 11.7 and $13.7 \mathrm{~s}$ on a Pentium $2.13 \mathrm{GHz}$ PC.

Apart from the IEEE 24 Bus Test System used in this case study we also applied the developed models to the 57 and 118 IEEE Bus Test Systems. For these two systems, we restrained the maximum allowable change on the variables of the linearized problem to $0.0001 \mathrm{pu}$ and the algorithm ran 88 and 136 iterations for operation cases similar to Case 3 described above. The corresponding CPU time was 49.9 and $502.8 \mathrm{~s}$. It should be referred that the level of congestion on these two systems was higher than on the IEEE 24 Bus Test System. This fact together with the reduction by 10 times of the specified allowable change of the variables of the linearized problem determines a larger number of iterations to get convergence and a larger CPU time when compared with the values indicated for the IEEE 24 Bus Test System.

\section{Conclusions}

In this paper we presented two new models aiming at remarrying active and reactive power while retaining competitive aspects of electricity markets. The development of these models and the related research work was suggested by the fact that electricity markets are typically based on a set of activities ran in terms of a chronologic sequence that, in some cases, can display several inefficiencies, namely due to the coupling between active and reactive power. In the developed models we addressed this issue by explicitly considering the capability diagram of synchronous 
generators defined in the PQ plane, the ac power flow equations and the branch thermal limit constraints expressed in terms of the apparent power. The developed approach is based on the Market Operator cleared quantities and on the Bilateral Contract injections and, if there are violated constraints, it aims at identifying a new operation point using generator and demand adjustment bids. This means that the System Operator will only change the initial schedule if that is required from a technical point of view. It is also important to refer two features of the developed models. They include generator adjustment variables related with the compensation of branch active losses and they are able to output the active and reactive nodal marginal prices. These features can contribute to design more robust tariff systems and as whole increase the realism and the potential applicability of this kind of models.

\section{Acknowledgements}

The first author wants to thank the PRODED III program, financed by EU and the FSE fund that supported this research.

\section{References}

[1] EU Commission and Parliament, EU 2003/54 Directive Establishing the Common Rules for the Internal Rules for the Internal Electricity Market, EU, June 2003

[2] M.H. Gomes, J.T. Saraiva, Active/reactive dispatch in competitive environment, in: Proceedings of the 2005 IEEE St. Petersburg Power Tech., St. Petersburg, Russia, 2005.

[3] N.H. Dandachi, M.J. Rawlins, O. Alsaç, M. Prais, B. Stott, OPF for reactive pricing studies on the NGC system, IEEE Trans. Power Syst. 11 (1996) 226-232.

[4] Y.-J. Zhang, Z. Ren, Optimal reactive power considering costs of adjusting the control devices, IEEE Trans. Power Syst. 20 (2005) 1349-1356.

[5] W.-H.E. Liu, X. Guan, Fuzzy constraint enforcement and control action curtailment in an optimal power flow, IEEE Trans. Power Syst. 11 (1996) 639-645.

[6] S. Hao, A. Papalexopoulos, Reactive power pricing and management, IEEE Trans. Power Syst. 12 (1997) 95-104.

[7] V. Dona, A. Paredes, Reactive power pricing in competitive electric markets using the transmission losses function, in: Proc. IEEE Porto Power Tech. Conference, Porto, Portugal, 2001.

[8] J. Zhong, E. Nobile, A. Bose, K. Bhattacharya, Localized reactive power markets using the concept of voltage control areas, IEEE Trans. Power Syst. 19 (2004) 1555-1561.
[9] J.W. Lamont, J. Fu, Cost analysis of reactive power support, IEEE Trans. Power Syst. 14 (1999) 890-898.

[10] G. Gross, S. Tao, E. Bompard, G. Chicco, Unbundled reactive support service: key characteristics and dominant cost component, IEEE Trans. Power Syst. 17 (2002) 283-289.

[11] S. Hao, A reactive power management proposal for transmission operators, IEEE Trans. Power Syst. 18 (2003) 1374-1381.

[12] B. Venkatesh, G. Sadasivam, M.A. Khan, A new optimal reactive power scheduling method for loss minimization and voltage stability margin maximization using successive multi-objective fuzzy LP technique, IEEE Trans. Power Syst. 15 (2000) 844-851.

[13] K. Papadogiannis, N. Hatziargyriou, J.T. Saraiva, Bid-based coupled active/reactive dispatch using simulated annealing, Int. J. Eng. Intell. Syst. 12 (2004) 175-183.

[14] S. Ahmed, G. Strbac, A method for simulation and analysis of reactive power market, IEEE Trans. Power Syst. 15 (2000) 1047-1052.

[15] E. Luiz da Silva, J. Hedgecock, J.C.O. Mello, J.C. Ferreira da Luz, Practical cost-based approach for the voltage ancillary service, IEEE Trans. Power Syst. 16 (2001) 806-812.

[16] K. Bhattacharya, J. Zhong, Reactive power as an ancillary service, IEEE Trans. Power Syst. 14 (2001) 294-300.

[17] J. Zhong, K. Bhattacharya, Toward a competitive market for reactive power, IEEE Trans. Power Syst. 17 (2002) 1206-1215.

[18] E.E. El-Araby, N. Yorino, Y. Zoka, Optimal procurement of VAR ancillary service in the electricity market considering voltage security, in: Proc. International Symposium on Circuits and Systems, ISCAS 2005, Kobe, Japan, 2005

[19] E.L. Miguélez, L.R. Rodriguez, T. Gomez San Roman, F.M.E. Cerezo, M.I.N. Fernández, R.C. Lafarga, G.L. Camino, A practical approach to solve power system constraints with application to the spanish electricity market, IEEE Trans. Power Syst. 19 (2004) 2029-2037.

[20] Task Force of Application of Probabilistic Methods Subcommittee, IEEE reliability test system, IEEE Trans. PAS PAS-98 (1979) 20472054.

Mário Helder Rodrigues Gomes was born in Mozambique in 1972. He received his licentiate and M.Sc. degrees from Faculdade de Engenharia da Universidade do Porto (FEUP) in 1997 and 2000 in electrical and computer engineering. In 1997 he joined the Polytechnic Institute of Tomar. He currently has a grant from the PRODED government financed program and he is completing his $\mathrm{Ph} . \mathrm{D}$. at FEUP.

João Tomé Saraiva was born in Porto, Portugal in 1962. In 1987, 1993 and 2002 he got his M.Sc., Ph.D., and Agregado degrees in electrical and computer engineering from the Faculdade de Engenharia da Universidade do Porto where he is currently professor. In 1985 he joined INESC Porto where he was head researcher or collaborated in several projects related with the development of DMS systems, quality in power systems, and tariffs due for the use of transmission and distribution networks. Several of these projects were developed under consultancy contracts with the Portuguese Electricity Regulatory Agency. 